\title{
Editorial: Can the Health Implications of Land-use Change Drive Sustainability?
}

In a whimsical moment, we wonder if other primates question whether humans are the most intelligent among them. Why has this species polluted the seas, rivers, air and is now destroying lands that are vital for its own existence and sustainability? Why is this process continuing despite having already driven the sixth mass extinction of life on Earth? For thousands of years, people have cut down forest to provide land for agriculture in a process that seemed sustainable. However, the last 70 years has seen anthropogenic land use change proceed at an unprecedented, exponentially accelerating scale. This process is especially severe in developing countries where logging, human settlements, and the building of roads and highways support a drive for increased agricultural production. These activities are often hugely profitable, contributing to a nation's economic success. But these short-term economic gains are sometimes marred by chronic negative externalities. The destruction of native forest, wetlands and mangroves reduces critical ecosystem services on which we depend: pollination of fruits and crops vital to a country's growing population, removal of fish nurseries and breakdown of water purification pathways. The impacts of deforestation on ecosystem services are significant for human well-being, in addition to our economic growth, and are used widely as an argument to reduce land use change.

In this Special Focus issue of EcoHealth, we highlight another critical implication of deforestation and land use change-the rise of infectious disease burdens in human and livestock populations. Over $30 \%$ of all emerging infectious diseases are driven by factors associated with land use change and agricultural development. This process leads to expansion of wildlife hunting and trade networks that are responsible for multiple outbreaks of Ebola virus, and the first pandemic of the twenty-first century-SARS. This last disease resulted in US \$10-50 billion in economic shock damages to the global trade and travel system. In a world that increasingly depends on globalized trade for economic stability, we ponder on the question-can we really afford these impacts? In the region we are focusing on in this issue-Southeast Asia-a smaller outbreak caused by Nipah virus in 1999 caused around US\$550-650 million. This was largely due to lost earnings from pig exports when the virus emerged in pig farms leading to $40 \%$ fatality in pig farm workers, and the closure of a new export industry for Malaysia. 20 years on from this event, we still feel its impacts on our national psyche and undoubtedly continue to suffer from economic losses as we adapt to the viral diversity that land use change unleashes.

Unfortunately, as if to confirm our primate relatives' worst fears, we seem to be repeating the process and driving new risks for our own health. Sabah on the island of Borneo is one of the 13 states of Malaysia and still has significant tracts of tropical forest, mangroves and wetlands. The drive for economic development is pushing new roads and expanding human settlements. While economic development and food security through agricultural expansion are vital, new data link these processes to outbreaks of dengue, tuberculosis, leptospirosis, Plasmodium malaria and melioidosis. In particular, zoonotic $P$. knowlesi malaria transmitted from macaques to people has been conclusively linked to land use change. Haze events drifting over from slash-and-burn agriculture on Indonesian Sumatra lead to respiratory infections, and school and office closures to avoid them. The rise in land use change will increase this. 
In this special focus issue, we ask the question: Is there evidence that the health impacts of land use change are important enough to drive a push for sustainability? The authors of this article believe it is. With growing public awareness of the direct impacts of forest burning on our health and a marked rise in concern about direct impacts of climate change, we believe that it is timely and critical. Demonstrating the linkages between health outcomes and deforestation may provide the ideal incentive that governments need to move to sustainable development in a region where the allure of rapid economic growth on the back of a chainsaw is hard to resist.

Kamruddin Ahmed

Borneo Medical and Health Research Centre and Department of Pathobiology and Medical Diagnostics, Faculty of Medicine and Health Sciences,

Universiti Malaysia Sabah, Kota Kinabalu, Sabah, Malaysia
Mohammad Saffree Jeffree

Department of Community and Family Medicine, Faculty of Medicine and Health Sciences,

Universiti Malaysia Sabah, Kota Kinabalu, Sabah, Malaysia

Tom Hughes, and Peter Daszak

EcoHealth Alliance, 460 West 34th Street - 17th Floor, New York, NY 10001, USA

e-mail: tom.hughes@ecohealthalliance.org

Published online: December 6, 2019 